\title{
Evaluation of Diagnostic Test in Emerging Carbapenem Resistant Gram Negative Bacilli in Patients admitted to Tertiary Care Centre in North India
}

\author{
Munesh Kumar Sharma ${ }^{1 *}$, Dakshina Bisht ${ }^{1}$ and Shekhar Pal ${ }^{2}$ \\ ${ }^{1}$ Department of Microbiology, Santosh Medical College, Ghaziabad, NCR Delhi, India \\ ${ }^{2}$ Department of Microbiology, Doon Medical College, Dehradun, India \\ *Corresponding author
}

\begin{tabular}{|l|}
\hline K e y w o r d s \\
B-lactam antibiotics, \\
Carbapenems, \\
Metallo beta \\
lacatamases, \\
Double disc \\
synergy test, \\
Meropenem \\
\hline Article Info \\
\hline Accepted: \\
17 March 2019 \\
Available Online: \\
10 April 2019 \\
\hline
\end{tabular}

Keywords

$\beta$-lactam antibiotics, Carbapenems,

Metallo beta lacatamases,

Double disc

synergy tes

Meropenem

Accepted:

10 April 2019

\section{A B S T R A C T}

Carbapenem antibiotics are very often used against multidrug resistant strains clinically troublesome pathogens which developed and proved that the resistance and metallo- $\beta$ lactamases (MBL) production were a disaster in treating infections. The identification and detection of MBL-producing bacterial strains were having crucial importance for the prevention of nosocomial infections. Therefore the present study was undertaken for screening MBL production Gram Negative bacteria. One hundred twenty two 122 consecutive Non-repetitive isolates of gram negative bacilli clinical isolates were subjected to susceptibility testing by disc-diffusion test on Mueller Hinton Agar. Meropenem resistant (MR) strains MBL production among MR stains were further screened by Meropenem- EDTA combined disc synergy test (M-CDST) and Meropenem-EDTA double-disc synergy test (M-DDST). A total of 31 isolates showed resistance to Meropenem which were screened and $29(93.55 \%)$ isolates gave positive result by MDDST whereas $27(87 \%)$ were MBL producers by M-CDST. Escherichia coli isolates recorded highest as MR strains were identified. For the treatment, implementation of effective infection control and prevention of nosocomial dissemination used the procedure for detection and identification of carbapenem resistant by most reliable method for study of MBLs produced isolates. The more effective method was M-DDST in comparison of other method as M-CDST.

\section{Introduction}

The emergence of carbapenem resistant strains among gram negative bacteria is a notable threat. Clinically relevant bacterial species detected often resistant to different $\beta$ lactam antibiotics, including the antibiotics which cover extended spectrum cephalosporins, but rarely to carbapenems
(Chu et al., 2001). Among the B-lactams drugs, carbapenems were potent agents for treatment of serious infections by gramnegative bacteria. Their broad spectrum activity and resistance to hydrolysis by most B-lactamases, including the extendedspectrum B-lactamases (ESBL) (Bush et al., 1995). Carbapenems antibiotics are the drug of choice for treatment of extended spectrum 
beta lactamase (ESBL) producing gram negative bacterial infections where the penicillin and other group cephalosporin antibiotics were resistant. Resistant Gram negative bacterial isolates are the important causative agents for urinary tract infections, bloodstream infections, healthcare-associated pneumonia, intra-abdominal infections and ventilator associated pneumonia. The increasing resistance of Carbapenem antibiotics in family Enterobacteriaceae and other group of Gram negative bacilli were a significant challenge with increasing prevalence. So Gram negative bacteria recognized resistance against different group of antibacterial drugs Worldwide. (NNIS, 2004)

However, in past few years resistance to carbapenems due to production of carbapenemases have been reported. Carbapenemases may be defined as betalactamases that significantly hydrolyze at least imipenem or meropenem. Most significant involved in acquired resistance are of Ambler molecular classes A, B and D. Class B or the Metallo-beta-lactamases (MBLs) enzymes are the most significant carbapenemases (Nordmann and Poirel, 2002; Deeba Bashir et al., 2011; Walsh et al., 2005). Carbapenemases especially MBLs due to transferrable in character are the most feared because it can hydrolyse almost all antibiotics including carbapenem antibiotics. So the study was conducted the detection of the carbapenem antibiotics resistance in gram negative bacilli isolates from different clinical specimens by phenotypic methods which may help to screen the population in hospital environment to guide effective empirical therapy.

\section{Materials and Methods}

A prospective study was carried out on 122 non repetitive gram negative bacilli isolates which has been isolated various clinical specimens from hospitalized patients. The study was approved by institutional ethics committee. The isolates were identified as per standard conventional methods as per CLSI guidelines 2010 (CLSI, 2010) in which incorporated the antibiotics were ampicillin (10 mcg), amocicillin/clavulenic acid (30 mcg) gentamicin (10 mcg), amikacin (30 $\mathrm{mcg})$, netilmicin (10 $\mathrm{mcg})$, cefotaxime (30 $\mathrm{mcg}$ ), ceftriaxone (30 mcg), ceftazidime (30 $\mathrm{mcg}$ ), cefepime (30 mcg), CIprofloxacin (5 $\mathrm{mcg})$, meropenem (10 $\mathrm{mcg})$, cefoperazone/ sulbactam (75/10 mcg), piperacillin/ tazobactum $(100 / 10 \mathrm{mcg})$ with polymyxin B (300 Units), tigecycline (15 mcg) and these were tested for in-vitro Carbapenem resistance and then tested to see MBL production in the bacterial isolates.

Isolated Gram negative bacilli identified from different clinical specimens which were resistant to carbapenem group of antibiotic as Meropenem. The gram-negative bacilli were showing the resistance for carbapenem antibiotic on routine screening was confirmed for presence of MBL production. Briefly, Muller-Hinton agar used for antibiotic susceptibility testing.

The combined disc and double disc synergy test methods were used to confirm above resistance mechanisms for $\mathrm{MBL}$ production (Lee et al., 2003).

\section{Meropenem-EDTA combined disc synergy test (CDST-Meropenem)}

Disks of Meropenem (10mcg, Himedia) and Meropenem with ethylene diamine tetraacetic acid, (EDTA) (10mcg $+750 \mathrm{mg}$, prepared in house) for MBL detection were used. Inoculated plates were incubated for 16-18 hours at $37^{\circ} \mathrm{C}$. If the increase in inhibition zone with Meropenem- EDTA disc was $\geq 7$ $\mathrm{mm}$ than the Meropenem disc alone then it was considered as MBL positive. 
Meropenem-EDTA Double Disc Synergy Test (DDST-Meropenem)

A Meropenem (10ug) disc was placed $20 \mathrm{~mm}$ center to center from a blank disc containing 10ul of $0.5 \mathrm{M}$ EDTA (750ug). Inoculated plates were incubated for $16-18$ hours at $37^{\circ} \mathrm{C}$. If enhancement in zone of inhibition between Meropenem and EDTA disc which was considered as positive for MBL production.

\section{Results and Discussion}

A total of 122 consecutive Non-repetitive isolates of gram negative bacilli obtained from various clinical samples were included in the study out of which 46 were isolated highest from pus, 32 follow urine, 21 sputum, 15 blood, 3 Urine Catheter tip, 2 Endotracheal Tube, 2 fluids and 1 from otitis media as depicted in Table 1.

Out of 122 Gram Negative Bacilli isolates were highest in pus $46(37.7 \%)$. Out of 31 carbapenem resistant isolates in pus identified highest isolates 11 (35.48\%), shown in Table 1. In 31 carbapenem resistant isolates, highest isolates were recorded in surgical ward $12(39 \%)$, followed medicine $7(23 \%)$, ICU $5(16 \%)$, OBS/Gynae 4(13\%), Orthopedic $2(6 \%)$ and Paediatric 1(3\%) shown in Chart no 1. Antimicrobial susceptibility in MBL producing bacterial strains showed resistant to different antibiotics as group of cephalosporins, aminoglycosides, fluoroquinolones, carbapenem drug as meropenem, and seen $100 \%$ sensitive for Polymyxin B, colistin followed $46 \%$ Tigecycline which were showed in Table 2. In Gram Negative Bacilli out of 31 carbapenem resistant highest isolate was Escherichia coli 8(25.81\%) followed Pseudomonas aeruginosa and Acinetobacter baumannii 6(19.35\%), Klebsiella pneumoniae 4(12.9\%), Klebsiella oxytoca 2(6.45\%), Proteus mirabilis 2(6.45\%), Other GNB 2(6.45\%) and
Citrobacter freundii 1(3.23\%). MBL detection test showed positive, for MBL production higher by DDST (29 isolates) and by CDST (27 isolates), shown in Table 3.

Infections caused by multidrug resistant gram negative bacterial where Carbapenem antibiotic proved most potent agents for treatment. MBL production is a most important mechanism to hydrolyse the Carbapenem antibiotics which emerged as the Carbapenem resistance. As per the therapeutic significance these bacterial isolates in study were also showing resistance for many other antibiotic groups like beta-lactams, aminoglycosides, fluoroquinolones and out of these, options left for therapy are use of Polymixin B and Colistin antimicrobial agent which carry potential toxicity (Gupta et al., 2012; Jesudason et al., 2005; Gupta et al., 2006). In the study highest number of resistance strains found from surgical department as similar (Nagaraj et al., 2012) except the other found in intensive care unit (Gupta et al., 2006; Mahajan et al., 2011; Sinha et al., 2007). The continuation in increasing prevalence of MBL producing strains has proved to be a clinical disaster and due to unnoticed spread within hospital or institution may turn to serious challenge for infection control management. And MBL producing strains may participate in horizontal MBL gene transfer to other pathogens in the hospital settings due to intrinsic capability of MBL producing strains. As Early detection of MBL producing bacteria in infections is need to treat appropriate with in time limit which might reduce the mortality when patient stay in hospital (Arakawa et al., 2000). MBL producing strain screening methods had been employed in different studies but due to no standard guidelines CLSI for detection of MBL which not laid Performance standards (Behera et al., 2008). In the present study we had used two conventional phenotypic tests 
for detection of MBL production as Meropenem-EDTA Combined Disc Test (CDST) and Meropenem-EDTA Double Disc Synergy Test (Meropenem-DDST). Although to see the Meropenem resistance by E test is also used for MBL detection but CDST and DDST are comparable to it and are also simple, reliable, inexpensive and reproducible (Yan et al., 2004). We had found that with Meropenem-EDTA DDST, the positives and negatives properly but with CDST it may be due to subjective variations with calculation for preparation of standard reagents. DDST identification was done with discriminating the true synergism. So, the DDST method using Meropenem-EDTA had good impact over CDST. As per the finding is in accordance with other studies which had found DDST to be one of the most sensitive technique for detecting MBL in comparison of CDST.

Table.1 Sample wise distribution of clinical isolates with carbapenem resistance

\begin{tabular}{|l|l|l|}
\hline Specimens & Clinical Isolates [no. $(\%)]$ & $\begin{array}{l}\text { Carbapenem Resistant isolates [no. } \\
(\mathbf{\%})]\end{array}$ \\
\hline Pus & $46(37.7)$ & $11(35.48)$ \\
\hline Urine & $32(26.23)$ & $8(25.81)$ \\
\hline Sputum & $21(17.21)$ & $5(16.13)$ \\
\hline Blood & $15(12.3)$ & $3(9.68)$ \\
\hline Urine Catheter Tip & $3(2.46)$ & $2(6.45)$ \\
\hline ET tube & $2(1.64)$ & $1(3.23)$ \\
\hline Fluid & $2(1.64)$ & $1(3.23)$ \\
\hline Otitis media & $1(0.82)$ & 0 \\
\hline Total & $\mathbf{1 2 2}$ & $\mathbf{3 1}$ \\
\hline
\end{tabular}

Table.2 In vitro available susceptibility of MBL and Non MBL producing GNB isolates

\begin{tabular}{|l|c|c|}
\hline Antibiotics & $\begin{array}{c}\text { Number of Non MBL Strain } \\
(\mathbf{n = 1 2 2})\end{array}$ & $\begin{array}{c}\text { Percentage of MBL Strains } \\
(\mathbf{n = 3 6})\end{array}$ \\
\hline Ampicillin & 30 & 0 \\
\hline Amoxicillin/Clavulanic Acid & 78 & 0 \\
\hline Cefotaxime & 112 & 0 \\
\hline Ceftriaxone & 114 & 0 \\
\hline Ceftazidime & 108 & 0 \\
\hline Cefepime & 116 & 0 \\
\hline Piperacillin/Tazobactam & 120 & 0 \\
\hline Gentamicin & 94 & 0 \\
\hline Amikacin & 114 & 0 \\
\hline Neticillin & 94 & 0 \\
\hline Ciprofloxacin & 114 & 0 \\
\hline Cefoperazone/Sulbactam & 92 & 0 \\
\hline Meropenem & 122 & 0 \\
\hline Tigecycline & 122 & 46 \\
\hline Colistin & 122 & 100 \\
\hline Polymyxin B & 122 & 100 \\
\hline
\end{tabular}


Table.3 Carbapenem resistant isolates with difference between MBL detection tests

\begin{tabular}{|c|c|c|c|}
\hline \multirow[t]{2}{*}{ Microorganism } & \multirow{2}{*}{$\begin{array}{c}\text { Carbapenem } \\
\text { Resistant Isolates }\end{array}$} & \multicolumn{2}{|c|}{ MBL detection test } \\
\hline & & $\begin{array}{c}\text { By DDST } \\
{[\mathrm{n}=29(\%)]}\end{array}$ & $\begin{array}{c}\text { By CDST } \\
{[\mathrm{n}=27(\%)]}\end{array}$ \\
\hline Escherichia coli & $8(25.81)$ & $8(27.59)$ & $7(23.93)$ \\
\hline Pseudomonas aeruginosa & $6(19.35)$ & $6(20.69)$ & $6(22.22)$ \\
\hline Acinetobacter baumannii & $6(19.35)$ & $5(17.24)$ & $5(18.52)$ \\
\hline Klebsiella pneumoniae & $4(12.9)$ & $4(13.79)$ & $3(11.11)$ \\
\hline Klebsiella oxytoca & $2(6.45)$ & $2(6.9)$ & $2(7.41)$ \\
\hline Proteus mirabilis & $2(6.45)$ & $2(6.9)$ & $2(7.41)$ \\
\hline Other GNB & $2(6.45)$ & $1(3.45)$ & $1(3.70)$ \\
\hline Citrobacter freundii & $1(3.23)$ & $1(3.45)$ & $1(3.70)$ \\
\hline & 31 & & \\
\hline
\end{tabular}

Chart.1 Ward-wise distribution of Meropenem resistant 31 Gram Negative bacterial isolates

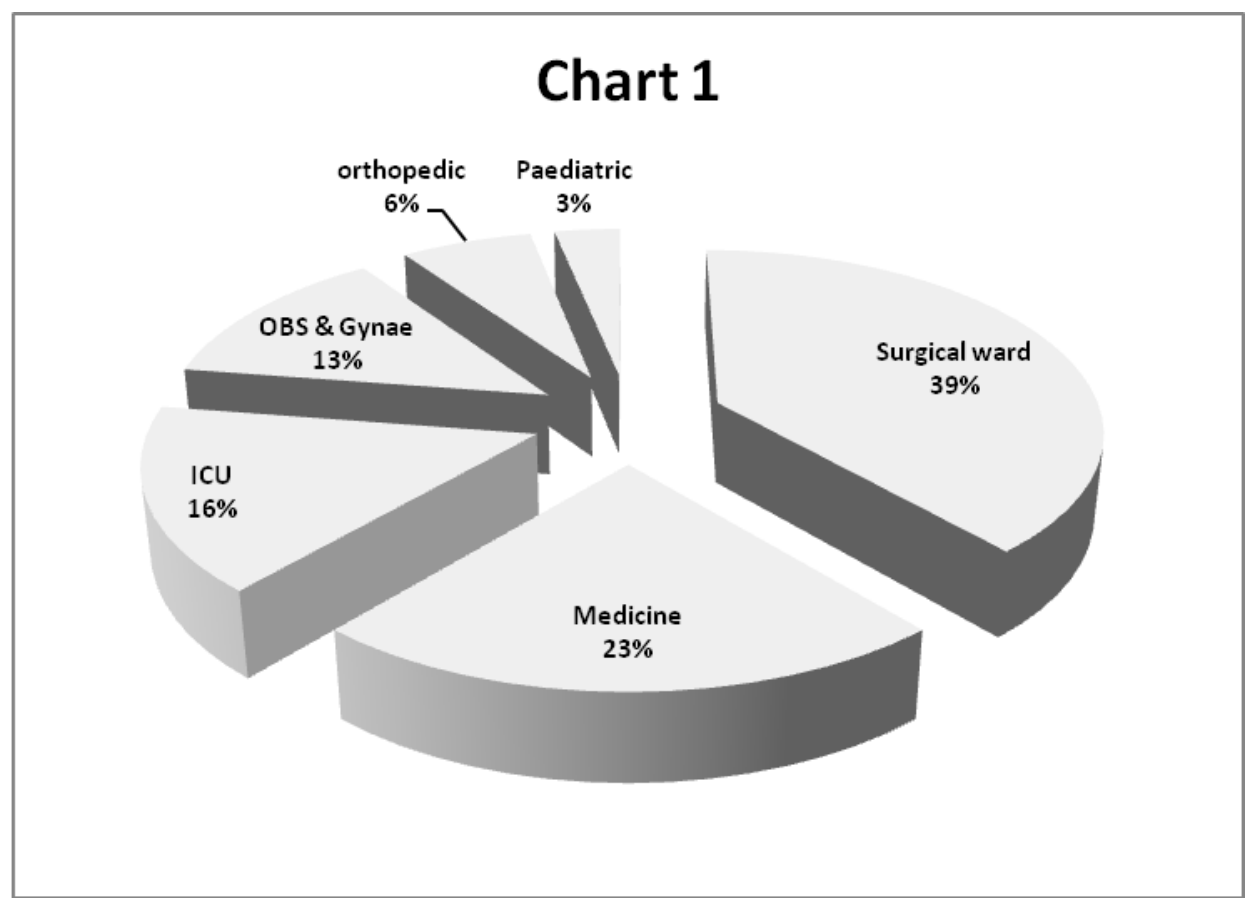


Fig.1 Showing antibiotic susceptibility testing in phenotypic method of Meropenem resistant strains for detection of metallo- $\beta$-lactamase producers. (A) Meropenem antibiotic resistant (B) Showing Combined Disc Synergy Test (Meropenem antibiotic incorporated EDTA disc) (C) Showing Double Disc Synergy Test (one disc Meropenem and other disc with EDTA) (D) Showing Blank disc

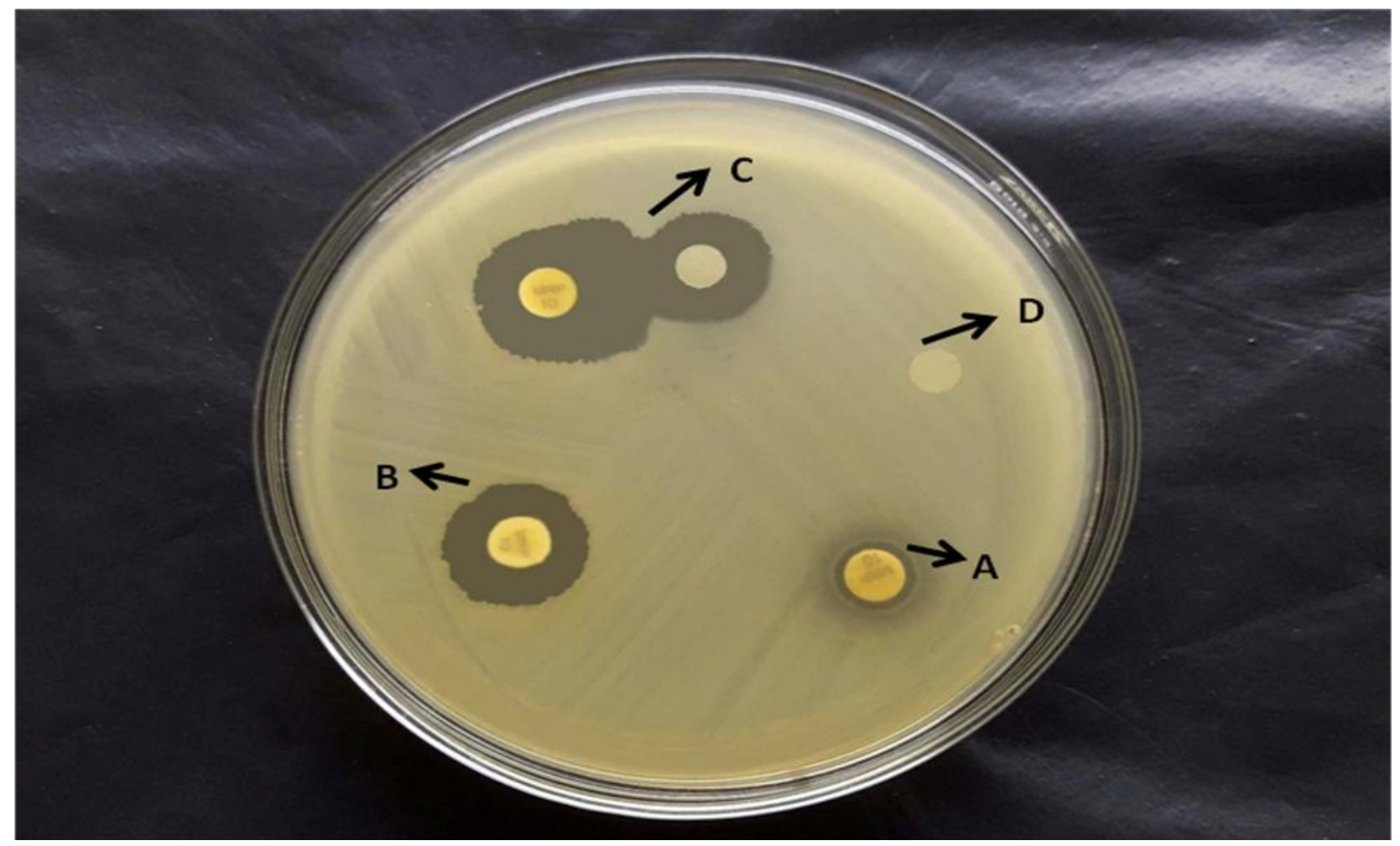

In India prevalence ranging from 14 to $20 \%$ has been reported in studies MeropenemDDST identified most sensitive test for detection of MBL production and hence Meropenem disc is a better option for screening MBL (Sinha et al., 2007; Sinha et al., 2013). In our study, out of 122 Gram Negative bacilli strains $31 \quad(25.41 \%)$ carbapenem resistant were prevalent. And out of 31 carbapenem resistant isolates DDST detected higher number of MBL producers 29 (93.55\%) than CDST 27 (87\%). In Figure 1 in vitro antibiotic susceptibility testing showing organism resistant to $10 \mu \mathrm{g}$ Meropenem (A), combined disc synergy test showing $\geq 7 \mathrm{~mm}$ increased size of zone of inhibition in Meropenem with EDTA combined disc (B), Double disc synergy test showing enhancement of zone of inhibition between
Meropenem and EDTA disc (C) and blank disc showing no zone of inhibition for microorganism which was used as a control (D).

In conclusion, metallo-beta lactamases producing GNB isolates disseminated worldwide. So study finds that antibiotic surveillance should be at regular interval in hospital settings. And strict Antibiotic policy enforcing judicious use of antibiotics in the different clinical departments for effective control of carbapenem resistant bacteria either patient stay is longer. There is a importance to introduce a simple, cheap, reliable and reproducible screening tests for early detection and identification of MBLproducing GNB in routine diagnostics laboratories. So we advise that in diagnostic 
procedure use additional EDTA disc $(750 \mu \mathrm{gm} / \mathrm{ml})$ on routine AST plates and also screen by Meropenem- DDST method for MBL producers.

\section{Acknowledgement}

I will be more thankful to technical staff for technical support and the M.Sc. medical students from microbiology department of Santosh Medical College.

\section{References}

Arakawa Y, Shibata N, Shibayama K, Kurokawa H (2000). Convenient test for screening metallo- betalactamases producing gram negative bacteria using thiol compounds. $J$ Clin Microbiol 38:40-3.

Behera B, Mathur P, Das A, Kapil A, Sharma $\mathrm{V}$ (2008). An evaluation of four different phenotypic techniquesfor Detection of metallo- $\beta$-lactamase producing Pseudomonas aeruginosa. Ind J Med Microbiol 26(3): 233-7.

Bush K, Jacoby GA, Medeiros AA (1995). A functional classification scheme for $b$ lactamases and its correlation with molecular structure. Antimicrobial Agents Chemother 39:1211-33.

Chu Y W, Shah M A, Houang E T S, Palepou M F I, Lyon D J, Woodford N et al., (2001). IMP-4 a novel metallo $\beta$ lactamase from nosocomial Acinetobacter spp. Collected in Hong Kong between 1994 and 1998. Antimicrob. Agents Chemother 45: 710714.

Clinical and Laboratory Standard Institute. Performance standards for antimicrobial susceptibility testing; Twentieth information supplement, document M100-S20. Warne, PA: Clinical Laboratory Standard Institute 2010, 45110.
Deeba bashir, Manzoor Ahmad Thokar,Basir Ahmad Fomda, Gulnaz Bashir, Danish Zaroor, Shabir Ahmad and Abubaker S. Toboli (2011). Detection of Metallo- $\beta$ lactamase (MBL) producing Pseudomonas aeruginosa at a tertiary care hospital Kashmir. Afr J Microbiol Res 5(2): 164-172.

Gupta E, Mohanty S, Sood S, Dhawan B (2006). Emerging resistance to Carbapenems in a tertiary care hospital in north India. Indian J Med Res 124(1): 95-8.

Gupta V, Sidhu S, Chander J (2012). Metallo- $\beta$ - lactamase producing nonfermentative gram-negative bacteria: An increasing clinical threat among hospitalized patients. Asian Pacific $J$ Trop Med 5(9):718-21.

Jesudason MV, Kandathil AJ, Balaji V (2005). Comparison of two methods to detect carbapenemase and metallo- $\beta$ lactamase production in clinical isolates. Indian J Med Res 121:780-3.

Lee K, Lim YS, Yong D, Yum JH, Chong Y (2003). Evaluation of the Hodge test and the imepenem-EDTA double-disk synergy test for differentiating metallo$\beta$-lactamase producing isolates of Pseudomonas spp. and Acinetobacter spp. J clin Microbial 41:4623-9.

Mahajan G, Sheemar S, Chopra S, Kaur J, Chowdhary D, Makhija S K (2011). Carbapenem resistance and phenotypic detection of carbapenemases in clinical isolates of Acinetobacter baumannii. Indian J Med Scienc 65(1):18-25.

Nagaraj S, Chandran SP, Shamanna P, Macaden R (2012). Carbapenem resistance among Escherichia coli and Klebsiella pneumoniae in a tertiary care hospital in south India. Indian $J$ Med Microbiol 30(1): 93-95.

National Nosocomial Infections surveillance (NNIS) System Report, data summary from January 1992 through June 2004, 
issued October 2004. Am J Infect Control 2004; 32: 470-85.

Nordmann P, Poirel L (2002). Emerging carbapenemases in gram negative aerobes. Clin. Microbiol Infect 8: 321333.

Sinha M, Srinivasa H (2007). Mechanisms of resistance to carbapenems in meropenem-resistant Acinetobacter isolates from clinical samples. Indian $J$ Med Microbiol 25(2):121-125.

Sinha N, Agarwal J, Srivastava S, M Singh (2013). Analysis of carbapenem- resistant Acinetobacter from a tertiary care setting in north India. Indian $\mathrm{J}$ Med Microbiol 31(1):60-63.

Walsh TR, Toleman MA, Poirel L, Nordman $P$ (2005). Metallo- $\beta$-lactamases: the quiet before storm? Clin Microbiol Rev 18: 306-325.

Yan JJ, Wu JJ, Tsai SH, Chuang CL (2004). Comparison of the double disk, combined-disk, and E Test methods for detecting metallo- $\beta$-lactamases in gramnegative bacilli. Diagn Microbiol Inf Dis 49: 5-11.

\section{How to cite this article:}

Munesh Kumar Sharma, Dakshina Bisht and Shekhar Pal. 2019. Evaluation of Diagnostic Test in Emerging Carbapenem Resistant Gram Negative Bacilli in Patients admitted to Tertiary Care Centre in North India. Int.J.Curr.Microbiol.App.Sci. 8(04): 2339-2346. doi: https://doi.org/10.20546/ijcmas.2019.804.273 